\title{
Astilbe chinensis Modulates Platelet Function via Impaired MAPK and PLC $\gamma 2$ Expression
}

\author{
Bo-Ra Jeon $\left(\mathbb{D},{ }^{1}\right.$ Muhammad Irfan, ${ }^{1}$ Seung Eun Lee, ${ }^{2}$ \\ Jeong Hoon Lee, ${ }^{2}$ and Man Hee Rhee $\mathbb{B}^{1}$ \\ ${ }^{1}$ Laboratory of Physiology and Cell Signaling, College of Veterinary Medicine, Kyungpook National University, \\ Daegu 41566, Republic of Korea \\ ${ }^{2}$ Department of Herbal Crop Research, National Institute of Horticultural and Herbal Science (NIHHS), \\ Eumseong 27709, Republic of Korea
}

Correspondence should be addressed to Man Hee Rhee; rheemh@knu.ac.kr

Received 28 February 2018; Accepted 25 July 2018; Published 5 August 2018

Academic Editor: Siew H. Gan

Copyright (c) 2018 Bo-Ra Jeon et al. This is an open access article distributed under the Creative Commons Attribution License, which permits unrestricted use, distribution, and reproduction in any medium, provided the original work is properly cited.

\begin{abstract}
Background. Platelets play major role in maintaining hemostasis while hyperactivation of platelets may lead to arterial thrombosis. Natural products and ethnomedicine have been shown to reduce the risk of cardiovascular diseases (CVDs). Astilbe chinensis is a perennial herb found in China, Korea, Russia, and Japan, which is also known for its medicinal effects, and has been used in Korean traditional medicine to treat inflammation, cancer, chronic bronchitis, and headache. We hypothesized that given herbal plant exhibits pharmacological activities against CVDs, and we specifically explored their effects on platelet function. Methodology. Platelet aggregation was evaluated using standard light-transmission aggregometry. Intracellular calcium mobilization was assessed using Fura-2/AM, and granule secretion (ATP release) was measured in a luminometer. Fibrinogen binding to integrin $\alpha_{\text {IIb }} \beta_{3}$ was assessed using flow cytometry. Phosphorylation of mitogen-activated protein kinase (MAPK) signaling molecules and activation of the phosphoinositide 3-kinase (PI3K)/Akt were assessed using western blots, and further, glycoprotein VI (GPVI) signaling components were studied using immunoprecipitation. Key Results. A. chinensis extracts potently and significantly inhibited platelet aggregation, calcium mobilization, granule secretion, and fibrinogen binding to integrin $\alpha_{\mathrm{IIb}} \beta_{3}$. Moreover, it significantly inhibited MAPK phosphorylation and expression of GPVI downstream signaling molecules. Conclusion. A. chinensis extract inhibited platelet aggregation and granule secretion and attenuated GPVI downstream signaling, indicating the potential therapeutic effects of this plant extract on the cardiovascular system and platelet function. We suggest that given plant extract may be a potent candidate to treat platelet-related CVDs and to be used as antiplatelet agent.
\end{abstract}

\section{Introduction}

Currently, cardiovascular diseases (CVDs) are the main cause of morbidity and mortality in developed countries [1]. There are multiple risk factors, but platelets, being a main etiological factor, play a central role in CVDs. Platelet aggregation is a key step in the development and progression of atherosclerotic plaques, which cause narrowing of the blood vessels that can ultimately lead to stroke and heart attack [2, 3]. Hyperactive platelets contribute to thrombosis and are important mediators of atherogenesis. Moreover, intravascular thrombosis is a factor that causes various CVDs. Pharmacological suppression of platelet function has shown great success in reducing thrombotic events, and a number of clinically approved antiplatelet drugs are available to treat cardiovascular ailments. However, these drugs can have serious complications (such as gastric bleeding) and are ineffective in some patients $[4,5]$, necessitating the need to develop effective and safer approaches to treat and prevent CVDs. One approach may include the use of natural products, like plant extracts, as antithrombotics and anticoagulants [6]. Currently, ethnomedicine and natural products are gaining interest as remedies for CVDs [7], as a number of dietary and herbal compounds have been shown to reduce the risk of CVDs [8].

Astilbe chinensis is a perennial herb found in China, Korea, Japan, and Russia and has been used in Korean traditional medicine as an analgesic and antipyretic $[9,10]$. Other 
traditional uses include anti-inflammatory, hepatoprotective, anticancer, and treatment of chronic bronchitis and headache [11]. However, cardiovascular effects of this herb have yet to be explored. To date, there is no report on the antiplatelet activity of $A$. chinensis. In this study, we evaluated the effects of this plant extract on the cardiovascular system, especially on platelet function, and explored the mechanistic aspects of their antiplatelet activities.

\section{Materials and Methods}

2.1. Chemicals and Reagents. Collagen, ADP, and thrombin were purchased from Chrono-log Corp. (Havertown, PA, USA). Fura-2/AM and dimethyl sulfoxide (DMSO) were obtained from Sigma-Aldrich (St. Louis, MO, USA). Fibrinogen Alexa Fluor ${ }^{\circledR} 488$ conjugate was purchased from Molecular Probes (Eugene, OR, USA), and the ATP assay kit was obtained from Biomedical Research Service Center (Buffalo, NY, USA). Antibodies against phospholipase C $\gamma 2$ (PLC $\gamma 2$ ), phospho-p44/42 (phospho-extracellular signalregulated kinase (ERK)), p44/42 (ERK), phospho-p38, p38, MEK, phospho-MEK, stress-activated protein kinase (SAPK)/c-Jun N-terminal protein kinase (JNK), phosphoSAPK/JNK, phospho-phosphoinositide 3-kinase (PI3K), PI3K, phospho-Akt, and Akt were acquired from Cell Signaling Technology (Beverly, MA, USA). Ultrapure water was obtained from J. T. Baker (Phillipsburg, NJ, USA). All chemicals were reagent grade.

2.2. Sample Preparation. Aerial part of A. chinensis plant was collected in Rural Development Administration (RDA) in Suwon city in 2004. The powder (100 g) of A. chinensis was extracted with methanol in accelerated solvent extraction system (Dionex, USA) at $50^{\circ} \mathrm{C}$ and evaporated in rotary evaporator (N-1000, Eyela, Japan). Finally, extract (17 g) from the powder of $A$. chinensis was obtained. Powder was dissolved in DMSO for further use in experiments. Vehicle concentration was kept at less than $0.1 \%$.

2.3. Animals. Male Sprague-Dawley (SD) rats (240-260 g) were purchased from Orient Co. (Seoul, Korea) and were acclimatized for one week before conducting the experiments in a special air conditioned animal room with $12 / 12 \mathrm{~h}$ light/ dark cycle at a temperature and humidity of $23 \pm 2^{\circ} \mathrm{C}$ and $50 \pm$ $10 \%$, respectively. All animal-related studies were carried out following the Institutional Animal Care and Use Committee (IACUC) guidelines, and the protocols were approved by the Ethics Committee of the College of Veterinary Medicine, Kyungpook National University, Daegu, Korea.

2.4. Platelet Preparation. Blood was collected from rats via heart puncture and transferred to a tube containing the anticoagulant, acid citrate dextrose (ACD) solution. Blood was centrifuged at $170 \times g$ for $7 \mathrm{~min}$ to obtain platelet-rich plasma (PRP). The PRP was further centrifuged at $350 \times g$ for $7 \mathrm{~min}$ to isolate platelets. The concentration of platelets was adjusted to $3 \times 10^{8}$ cells $/ \mathrm{mL}$ using Tyrode's buffer without calcium (137 mM NaCl, $12 \mathrm{mM} \mathrm{NaHCO}, 5.5 \mathrm{mM}$ glucose, $2 \mathrm{mM} \mathrm{KCl}, 1 \mathrm{mM} \mathrm{MgCl}$, and $1 \mathrm{mM} \mathrm{NaHPO}_{4}, \mathrm{pH}$ 7.4), and these platelets were used for aggregation assays. All platelet preparation procedures were performed at room temperature $\left(23 \pm 2^{\circ} \mathrm{C}\right)$.

2.5. Platelet Aggregation Assay and Scanning Electron Microscopy Analysis. Platelet aggregation was performed using a standard technique, light-transmission aggregometry (Chrono-log Corp., Havertown, PA, USA), as previously described [12]. Briefly, washed platelets were preincubated with various concentrations of $A$. chinensis extract or vehicle for $2 \mathrm{~min}$ at $37^{\circ} \mathrm{C}$ in the presence of $1 \mathrm{mM} \mathrm{CaCl}_{2}$, followed by stimulation with the agonist, collagen, ADP, or thrombin. The mixture was incubated for 5 min with continuous stirring.

A field emission scanning electron microscope (SU8220, Hitachi) was used to assess extent of platelet aggregation with ultrastructure image and platelet shape change at the Center for Scientific Instrument, Kyungpook National University, Daegu, Korea. Briefly, following the platelet aggregation assay, scanning electron microscopy was performed on collagenstimulated platelets pretreated with various concentrations of A. chinensis extract: (a) Resting, (b) Vehicle, (c) $25 \mu \mathrm{g} / \mathrm{mL}$, (d) $50 \mu \mathrm{g} / \mathrm{mL}$, and (e) $100 \mu \mathrm{g} / \mathrm{mL}$. The platelet mixture was fixed with $0.5 \%$ paraformaldehyde (first fixation) and osmium tetroxide (second fixation), dehydrated with various concentrations of ethanol, freeze-dried, and scanned.

2.6. Intracellular Calcium Ion Concentration $\left(\left[\mathrm{Ca}^{2+}\right]_{i}\right) \mathrm{Mea-}$ surements. The $\left[\mathrm{Ca}^{2+}\right]_{i}$ was assessed using Fura-2/AM as previously described [13]. Briefly, platelets were preincubated with $5 \mu \mathrm{M}$ Fura-2/AM for $1 \mathrm{~h}$ at $37^{\circ} \mathrm{C}$. Following incubation, the platelets were washed and treated with $A$. chinensis extract for $1 \mathrm{~min}$ in the presence of $1 \mathrm{mM} \mathrm{CaCl}_{2}$ at $37^{\circ} \mathrm{C}$, followed by stimulation with collagen for $2 \mathrm{~min}$. Fluorescence was recorded using a spectrofluorometer (F-2500, Hitachi, Japan) and $\left[\mathrm{Ca}^{2+}\right]_{i}$ was calculated with Schaeffer and Blaustein's method [14], using the following formula: $\left[\mathrm{Ca}^{2+}\right]_{i}$ in cytosol $=224 \mathrm{nM} \times\left(F-F_{\min }\right) /\left(F_{\max }-F\right)$, where $224 \mathrm{nM}$ is the dissociation constant of the Fura-2-Ca ${ }^{2+}$ complex and $F_{\text {min }}$ and $F_{\max }$ represent the fluorescence intensity levels at very low and very high $\mathrm{Ca}^{2+}$ concentrations, respectively.

2.7. ATP Release Assay. Washed platelets were preincubated with $A$. chinensis extract in the presence of $1 \mathrm{mM} \mathrm{CaCl}_{2}$ for $2 \mathrm{~min}$ at $37^{\circ} \mathrm{C}$ and then were stimulated with collagen for 5 min. The aggregation reaction was terminated and the platelet mixture centrifuged. The supernatant was used to measure ATP secretion with a luminometer (GloMax 20/20, Promega, Madison, WI, USA), using an ATP assay kit (Biomedical Research Service Center).

2.8. Flow Cytometry. The fibrinogen binding to integrin $\alpha_{\text {IIb }} \beta_{3}$ was quantified using flow cytometry as previously described [15]. Briefly, washed platelets were preincubated with $A$. chinensis extract for $2 \mathrm{~min}$ in the presence of $0.1 \mathrm{mM}$ $\mathrm{CaCl}_{2}$, followed by stimulation with collagen for $5 \mathrm{~min}$. The stimulated platelets were treated with fibrinogen Alexa Fluor ${ }^{\circledR}$ 488 conjugate $(20 \mu \mathrm{g} / \mathrm{mL})$ for $5 \mathrm{~min}$ at room temperature $\left(23 \pm 2^{\circ} \mathrm{C}\right)$ and were then fixed with $0.5 \%$ paraformaldehyde for $30 \mathrm{~min}$ at $4^{\circ} \mathrm{C}$. Following fixation, platelets were washed 
thrice in phosphate-buffered saline (PBS). Fluorescence was recorded with a BD FACSCalibur ${ }^{\mathrm{TM}}$ flow cytometer (BD Biosciences, San Jose, CA, USA), and data were analyzed using CellQuest $^{\mathrm{TM}}$ software (Becton Dickinson Immunocytometry Systems, San Jose, CA, USA).

2.9. Immunoprecipitation. Platelets were suspended at $8 \times 10^{8}$ cells/mL in modified Tyrode's-HEPES buffer for immunoprecipitation assays, and platelet aggregation was performed as described above. The reaction was terminated using an equal volume of ice-cold NP40 lysis buffer $\left(2 \%\right.$ [v/v] Nonidet ${ }^{\mathrm{TM}} \mathrm{P}-$ 40, $50 \mathrm{mM}$ Tris, $20 \mathrm{mM} \mathrm{NaF}, 25 \mathrm{mM} \beta$-glycerophosphate, 120 $\mathrm{mM} \mathrm{NaCl}, 10 \mathrm{mM}$ ethylenediaminetetraacetic acid [EDTA], 1 $\mathrm{mM}$ phenylmethylsulfonyl fluoride [PMSF], $2 \mathrm{mM} \mathrm{Na}_{3} \mathrm{VO}_{4}$, $5 \mathrm{mg} / \mathrm{mL}$ leupeptin, $5 \mathrm{mg} / \mathrm{mL}$ aprotinin, $1 \mathrm{mg} / \mathrm{mL}$ pepstatin $\mathrm{A}$, and $1 \mathrm{mM}$ benzamide, $\mathrm{pH}$ 7.5). The lysate obtained was precleared with protein A-sepharose (PAS) for $1 \mathrm{~h}$ at $4^{\circ} \mathrm{C}$. PAS was then removed and the lysate treated with pulldown antibody overnight at $4^{\circ} \mathrm{C}$ on a rotary shaker. Protein/antibody complexes were isolated with PAS for $4 \mathrm{~h}$ at $4^{\circ} \mathrm{C}$ and washed five times with lysis buffer. The beads were boiled in Laemmli sample buffer (2\% sodium dodecyl sulfate [SDS], $1 \% \beta$-mercaptoethanol, $0.008 \%$ bromophenol blue, $80 \mathrm{mM}$ Tris/HCl pH 6.8, and $1 \mathrm{mM}$ EDTA), and the proteins resolved using SDS-polyacrylamide gel electrophoresis (SDS-PAGE). The resolved proteins were transferred to polyvinylidene difluoride (PVDF) membranes; the membranes were blocked with $5 \%$ milk, probed with respective primary and secondary antibodies, and visualized using enhanced chemiluminescence (Advansta, CA, USA).

2.10. Immunoblotting. Platelet aggregation was terminated by the addition of lysis buffer (PRO-PREP; iNtRON Biotechnology, Seoul, Korea) to the mixture, followed by the estimation of protein concentration using the BCS assay (PROMEASURE; iNtRON Biotechnology, Seoul, Korea). Total platelet proteins were separated using 10\% SDS-PAGE and transferred to PVDF membranes. Membranes were blocked with $5 \%$ skim milk, probed with respective antibodies, and visualized using enhanced chemiluminescence (Advansta, CA, USA).

2.11. Statistical Analysis. Data were analyzed by one-way analysis of variance (ANOVA), followed by Dunnett's post hoc tests to measure statistical significance among and between measurements (SAS Institute Inc., Cary, NC, USA). All data are presented as the means \pm standard errors of the mean (SEMs). A $p$ value of 0.05 or less was considered statistically significant.

\section{Results}

3.1. A. chinensis Inhibits Collagen-Induced Platelet Aggregation. Initial screening with different agonists showed that $A$. chinensis extract inhibits collagen-induced platelet aggregation (Figure 1(a)). A significant and concentration-dependent inhibition of platelet aggregation was observed in platelets pretreated with $A$. chinensis extract compared with that of vehicle control (Figure 1(b)). Activation of platelet receptors (e.g., GPVI or P2Y12) triggers downstream signaling events which causes granule secretion, platelet shape change, and fibrin formation, subsequently leading to platelet aggregation. The continuous change in shape from the unactivated to the fully activated platelet is best seen on scanning electron microscopy. We confirmed these results using scanning electron microscopy to show inhibition of platelet shape change and platelet aggregation. A clear inhibition in platelet shape change can be seen in collagen-stimulated platelets pretreated with increasing concentrations of $A$. chinensis as compared with vehicle stimulated platelets (Figure 1(c)).

3.2. A. chinensis Reduces $\left[\mathrm{Ca}^{2+}\right]_{i}$ Mobilization and ATP Secretion. Calcium mobilization was assessed in platelets pretreated with $A$. chinensis extract or vehicle, and the results showed significant, concentration-dependent inhibition in intracellular calcium elevation, which indicates that $A$. chinensis extract may inhibit platelet aggregation by inhibiting calcium mobilization (Figure 2(a)). Granule secretion plays an important role in platelet activation and enhances platelet aggregation. ATP release was assessed in collagen-stimulated platelets pretreated with $A$. chinensis extract or vehicle. $A$. chinensis extract was found to inhibit ATP secretion in a significant manner (Figure 2(b)).

3.3. A. chinensis Inhibits Fibrinogen Binding to Integrin $\alpha_{I I b} \beta_{3}$. Fibrinogen binding to integrin $\alpha_{\mathrm{IIb}} \beta_{3}$ causes a conformational change in integrin $\alpha_{\mathrm{IIb}} \beta_{3}$ structure, which is also known as inside-out signaling, consequently leading to platelet aggregation. Our extract showed significant and dose dependent inhibition of fibrinogen binding to integrin $\alpha_{\mathrm{IIb}} \beta_{3}$ (Figure 3).

3.4. A. chinensis Reduces Phosphorylation of MAPK and MEK Signaling Molecules and PI3K/Akt Activation. Previous studies have shown that the MAPKs, ERK, JNK, and p38 are highly expressed in agonist-induced activated platelets and play vital roles in platelet activation [16]. A. chinensis extract significantly inhibited phosphorylation of all MAPK molecules in a concentration-dependent manner (Figure 4(a)). MEK is an upstream molecule in the ERK pathway [17], and A. chinensis extract significantly and concentrationdependently inhibited phosphorylation of this protein (Figure $4(\mathrm{a}))$.

The PI3K/Akt pathway has been shown to play an important role in platelet aggregation [18]. Therefore, we assessed activation of this pathway in collagen-stimulated platelets pretreated with $A$. chinensis extract and found that our extract had significant inhibitory potential against the activation of this pathway (Figure 4(b)). These results show that $A$. chinensis extract potentially inhibits platelet aggregation by inhibiting phosphorylation of MAPK and MAPKK signaling proteins and PI3K/Akt pathway activation.

3.5. A. chinensis Inhibits Phosphorylation of Proteins Involved in GPVI Signaling. The proteins $\mathrm{P} 13 \mathrm{~K}$ and phospholipase $\mathrm{C} \gamma 2$ (PLC $\gamma 2$ ) play significant roles in GPVI signaling and are crucial contributors to downstream outcomes, such as 


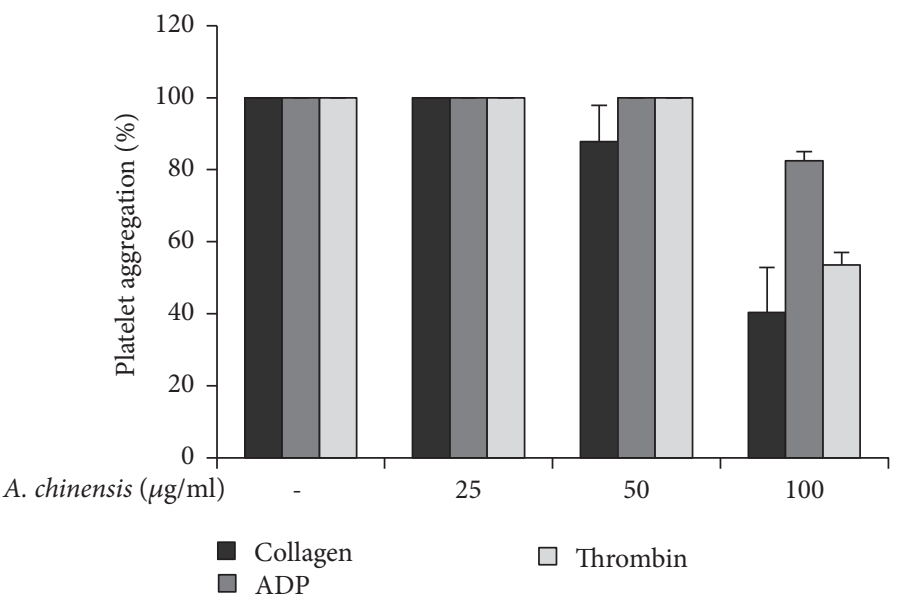

(a)

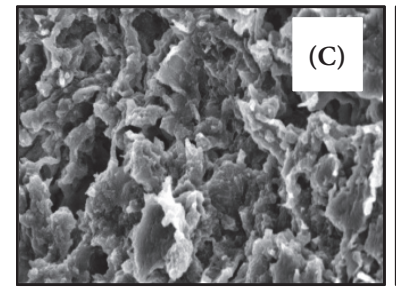

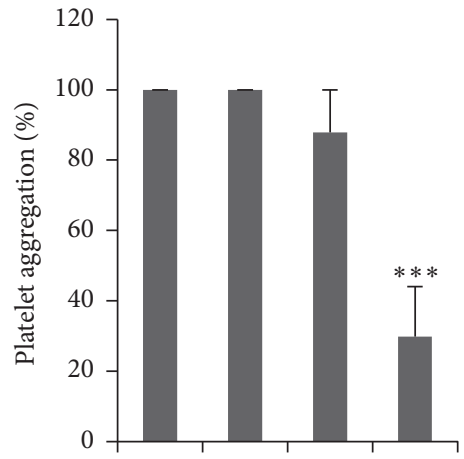

Collagen $(2.5 \mu \mathrm{g} / \mathrm{ml})++\quad+\quad+$ A. chinensis $(\mu \mathrm{g} / \mathrm{ml}) \quad-\quad 25 \quad 50 \quad 100$

(b)

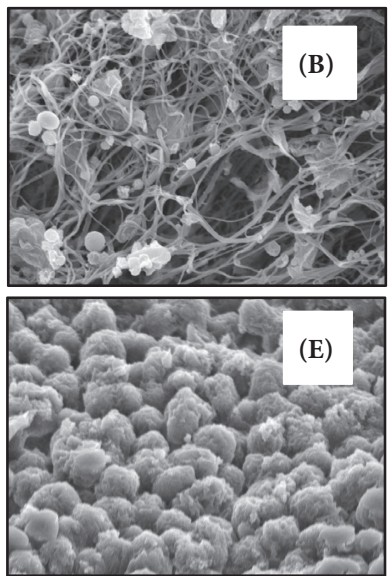

(E)

(c)

FIgURE 1: Astilbe chinensis extract inhibits collagen-stimulated platelet aggregation. (a-c) Washed platelets were pretreated with A. chinensis extract or vehicle for $2 \mathrm{~min}$ in the presence of $1 \mathrm{mM} \mathrm{CaCl}$, and then stimulated with collagen for $5 \mathrm{~min}$. (c) After the platelet aggregation reaction, scanning electron microscopy was performed. Representative scanning electron microscopy images of collagen $(2.5 \mu \mathrm{g} / \mathrm{mL})$ stimulated platelets pretreated with various concentrations of $A$. chinensis extract [(A) Resting, (B) Vehicle, (C) $25 \mu \mathrm{g} / \mathrm{mL}$, (D) $50 \mu \mathrm{g} / \mathrm{mL}$, and $(\mathrm{E}) 100 \mu \mathrm{g} / \mathrm{mL}]$. The graph represents the means \pm SEMs of at least four independent experiments. $* * * p<0.001$ versus control.

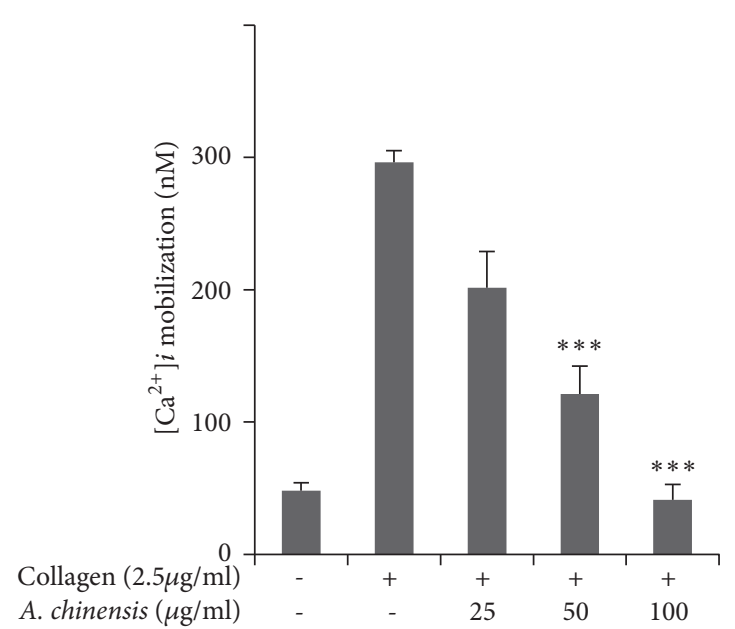

(a)

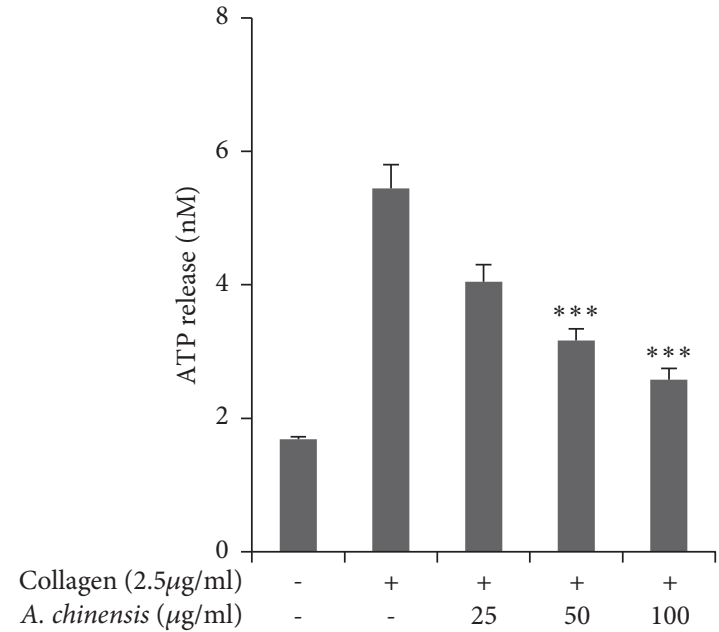

(b)

FIgURE 2: The inhibitory effect of Astilbe chinensis on collagen-stimulated $\left[\mathrm{Ca}^{2+}\right]_{i}$ elevation and ATP secretion. (a) Washed platelets were loaded with a calcium fluorophore $\left(5 \mu \mathrm{M}\right.$, Fura-2/AM) for $1 \mathrm{~h}$. Fura $2 / \mathrm{AM}$-loaded platelets were pretreated with $A$. chinensis for 2 min at $37^{\circ} \mathrm{C}$ and stimulated with collagen. (b) After platelet aggregation was terminated, the concentration of ATP was assessed in collagen-stimulated platelets treated with $A$. chinensis extract, using a luminometer. The results represent the mean \pm SEM of at least four independent experiments. $* * * p<0.001$ versus control. 


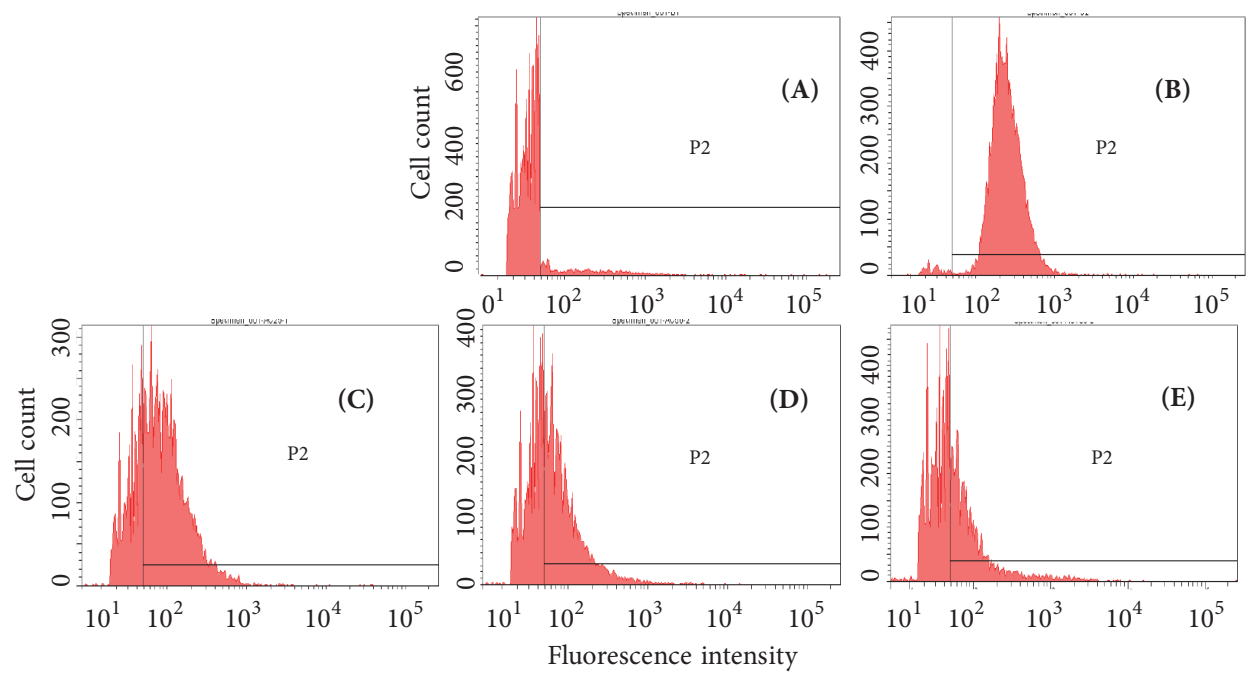

(a)

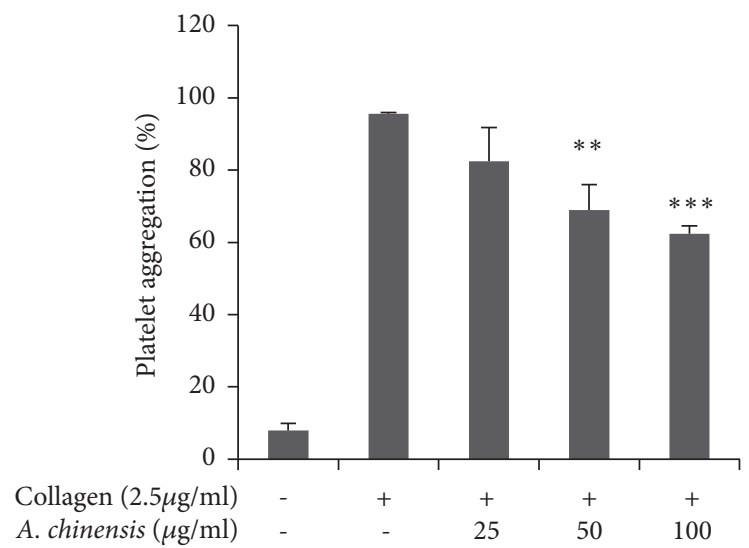

(b)

FIGURE 3: Astilbe chinensis extract blocks fibrinogen binding to integrin $\alpha_{\mathrm{IIb}} \beta_{3}$ in collagen-induced platelets. (a) Flow cytometry was used to measure fibrinogen binding to collagen $(2.5 \mu \mathrm{g} / \mathrm{mL})$-stimulated platelets pretreated with various concentrations of $A$. chinensis extract [(A) Resting, (B) Vehicle, (C) $25 \mu \mathrm{g} / \mathrm{mL}$, (D) $50 \mu \mathrm{g} / \mathrm{mL}$, and (E) $100 \mu \mathrm{g} / \mathrm{mL}$. (b) Bar graph summarizing the inhibitory effect of $A$. chinensis extract on fibrinogen binding to integrin $\alpha_{\mathrm{IIb}} \beta_{3} . * * \mathrm{p}<0.01$ and $* * * p<0.001$ versus control.

granule secretion and platelet aggregation [19]. Therefore, we examined the effect of $A$. chinensis extract on these proteins in the GPVI pathway. Our results show that A. chinensis extract markedly blocked interactions between the two proteins (Figure 5).

\section{Discussion}

Platelets are small fragments derived from megakaryocytes that help to maintain hemostasis and prevent blood loss by inducing fibrin clot formation at the site of vascular injury. However, hyperactivation of platelets may prove fatal and contributes to the formation of atherosclerotic plaques within blood vessels, which progressively restricts blood flow, leading to hypoxia and ischemic injury. Plaques can also rupture from vessels making emboli, leading to stroke and myocardial infarction [20]. A number of antiplatelet drugs are available and have proven to be beneficial in reducing the risk of thrombotic events. However, these drugs can produce undesirable side effects and complications, and they are ineffective in some patients [21], which necessitates the development of safer approaches to treat CVDs. Natural compounds have been shown to reduce CVDs [8], and, in our effort to discover alternative compounds, we found $A$. chinensis. These medicinal plants have been used in China, Korea, and Japan in traditional remedies $[11,22]$.

In the present study, we evaluated the inhibitory effects of $A$. chinensis extract on platelet aggregation and explored the mechanistic aspects of these extracts on platelet function. These extracts regulated $\left[\mathrm{Ca}^{2+}\right]_{i}$ mobilization and inhibited ATP secretion. Moreover, fibrinogen binding to integrin $\alpha_{\text {IIb }} \beta_{3}$ was inhibited, and activation of the MAPK and PI3k/Akt pathways was attenuated. Our results show that A. chinensis extract significantly inhibited $\left[\mathrm{Ca}^{2+}\right]_{i}$ mobilization in a concentration-dependent manner, and dense granule secretion. Intracellular calcium plays a critical role 


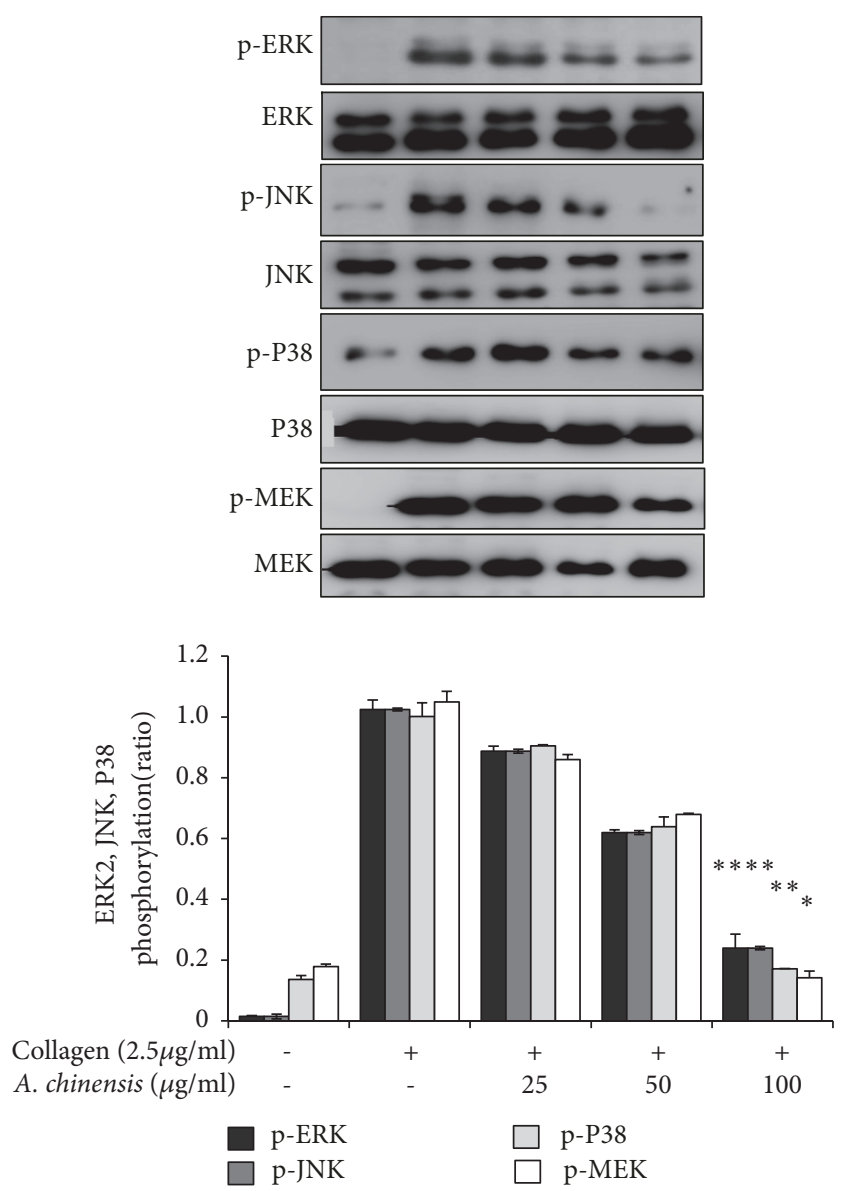

(a)
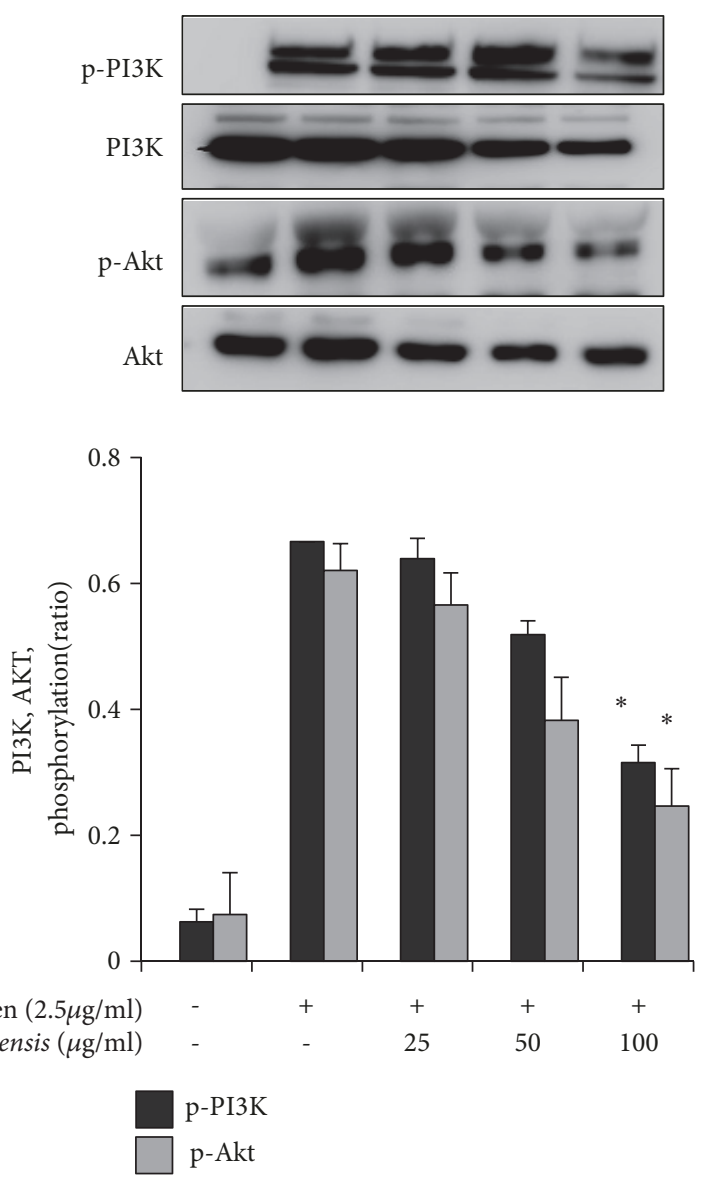

(b)

FIGURE 4: Astilbe chinensis extract attenuates mitogen-activated protein kinases (MAPKs) and MEK (a), and phosphoinositide 3-kinase (PI3K)/Akt (b) phosphorylation in collagen-stimulated platelets. Washed platelets were pretreated with A. chinensis extract and stimulated with collagen. After the reaction was terminated, protein was extracted from the platelets and analyzed for the phosphorylation of the indicated proteins by immunoblot analysis. Representative immunoblot images are shown, with the quantified data plotted below (mean \pm SEM, $\mathrm{n}=$ 3). $* p<0.05$ and $* * p<0.01$ versus the agonist-treated group.

in platelet activation, aggregation, and thrombus formation [23]. Increased $\left[\mathrm{Ca}^{2+}\right]_{i}$ triggers granule, which enhances platelet activation, while calcium chelation inhibits ATP release (dense granule secretion) [24]. Comparing our results with those in the literature, our data suggest that $A$. chinensis extract exerts inhibitory effects on granule secretions to regulate platelet function.

Activation of platelets leads to conformational changes in the structure of integrin $\alpha_{\mathrm{IIb}} \beta_{3}$, which enhances platelet aggregation [25]. Therefore, integrin inactivation is of great interest in the development of antiplatelet therapy. Altered fibrinogen binding to integrin $\alpha_{\text {II }} \beta_{3}$ in response to these conformational changes is also known as inside-out signaling, and later steps involve further signal transduction that is necessary for complete platelet aggregation [26]. Our results show that fibrinogen binding to integrin $\alpha_{\mathrm{IIb}} \beta_{3}$ was significantly inhibited by pretreatment of platelets with A. chinensis extract, which indicates that pretreatment of platelets with these extracts may attenuate the conformational changes (i.e., inside-out signaling) and impair integrin $\alpha_{\mathrm{IIb}} \beta_{3}$ activation.
Many studies have shown that platelets are continuously exposed to several factors that cause their activation and aggregation, such as collagen, ADP, thrombin, fibrinogen, von Willebrand factor (vWF), and thromboxane; some factors are also inhibitory, such as prostacyclin $\left(\mathrm{PGI}_{2}\right)$ and ADPase [27]. Any imbalance in these opposing factors may cause impairment in hemostasis; thus, a strong equilibrium is necessary for normal platelet function. Our results indicate that pretreatment of platelets with $A$. chinensis extract may contribute to maintaining this balance and hemostasis.

Platelets express MAPK that include ERK, JNK, and p $38^{\mathrm{MAPK}}$, which are activated by several agonists (e.g., collagen, ADP, and thrombin) [16]. ERK2 has been shown to enhance collagen-stimulated platelet secretion, while p38 is involved in platelet spreading and adhesion. ERK2 and p38 inhibitors have been shown to suppress platelet activation [28]. PI3K and PLC $\gamma 2$ play important roles in collagenstimulated GPVI downstream signaling and are involved in platelet granule secretion and aggregation [29]. Previous studies have shown that the PI3K/Akt pathway is involved 


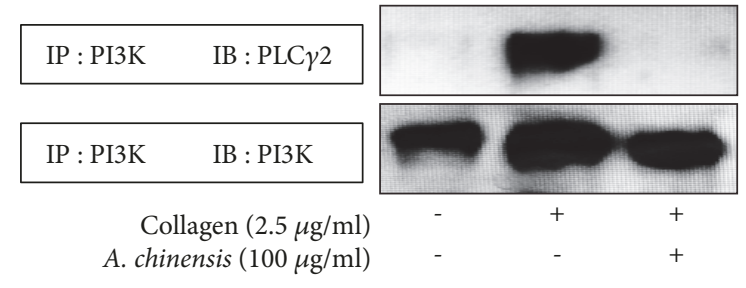

Figure 5: Astilbe chinensis extract attenuates collagen-stimulated phospholipase $\mathrm{C} \gamma 2$ (PLC $\gamma 2$ ) activation in the glycoprotein VI (GPVI) signaling pathway. Washed platelets were preincubated with $A$. chinensis extract or vehicle for $3 \mathrm{~min}$ and then were stimulated with collagen for $5 \mathrm{~min}$. Lysates $(100 \mu \mathrm{g} / \mathrm{mL})$ from A. chinensis extract-treated platelets were immunoprecipitated by incubating overnight with anti-PI3K antibody and then incubating with protein A-sepharose for $4 \mathrm{~h}$ at $4^{\circ} \mathrm{C}$. Immunoprecipitated proteins were separated by SDS-PAGE and immunoblotted to detect PLC $\gamma 2$ expression.

in cardiac protection by inducing antiapoptotic effects and reducing myocardial ischemia reperfusion injury (MI/RI) [30]. Moreover, studies have shown that platelet aggregation can be reversed in collagen- or ADP-stimulated platelets by blocking PI3K [31].

Our results show that $A$. chinensis extract inhibited the phosphorylation of these molecules, indicating that MAPK proteins and the PI3K/Akt pathway may be involved in the antiplatelet mechanism of $A$. chinensis extract. Further, $A$. chinensis extracts inhibited PI3K and PLC $\gamma 2$ interaction, indicating their possible mechanism of action in inactivating GPVI receptor downstream signaling. Moreover, our findings suggest that $A$. chinensis extract inhibits PI3K/Akt signaling and its involvement in modulating the reperfusion injury salvage kinase (RISK) pathway.

\section{Conclusion}

Our results show that $A$. chinensis extract is potent inhibitor of collagen-induced platelet aggregation, granule secretion, and fibrinogen binding to integrin $\alpha_{\mathrm{IIb}} \beta_{3}$. Moreover, the extract modulates platelet function via impaired MAPK phosphorylation and inactivation of PI3K/Akt pathway, suggesting their antiplatelet potential. We suggest that $A$. chinensis extract is potent antithrombotic candidate that can be used to treat platelet-related cardiovascular disorders.

\section{Data Availability}

All data generated or analyzed during this study are included in this published article.

\section{Disclosure}

The present data are part of Ph.D. dissertation for Bo-Ra Jeon.

\section{Conflicts of Interest}

The authors declare no conflicts of interest.

\section{Acknowledgments}

This research was supported by a grant from the National Research Foundation of Korea funded by the Korean government (Grant no. 2015R1D1A1A09057204).

\section{References}

[1] F. Maione, V. de Feo, E. Caiazzo, L. de Martino, C. Cicala, and N. Mascolo, "Tanshinone IIA, a major component of Salvia milthorriza Bunge, inhibits platelet activation via Erk-2 signaling pathway," Journal of Ethnopharmacology, vol. 155, no. 2, pp. 1236-1242, 2014.

[2] J. N. George, "Platelets," The Lancet, vol. 355, no. 9214, pp. 1531$1539,2000$.

[3] J. B. Michel, J. L. Martin-Ventura, A. Nicoletti, and B. Ho-TinNoé, "Pathology of human plaque vulnerability: mechanisms and consequences of intraplaque haemorrhages," Atherosclerosis, vol. 234, no. 2, pp. 311-319, 2014.

[4] N. Mackman, "Triggers, targets and treatments for thrombosis," Nature, vol. 451, no. 7181, pp. 914-918, 2008.

[5] N. E. Barrett, L. Holbrook, S. Jones et al., "Future innovations in anti-platelet therapies," British Journal of Pharmacology, vol. 154, no. 5, pp. 918-939, 2008.

[6] S. Rastogi, M. M. Pandey, and A. K. S. Rawat, "Traditional herbs: a remedy for cardiovascular disorders," Phytomedicine, vol. 23, no. 11, pp. 1082-1089, 2016.

[7] J. M. Kerver, E. J. Yang, L. Bianchi, and W. O. Song, "Dietary patterns associated with risk factors for cardiovascular disease in healthy US adults," American Journal of Clinical Nutrition, vol. 78, no. 6, pp. 1103-1110, 2003.

[8] A. K. Dutta-Roy, "Dietary components and human platelet activity," Platelets, vol. 13, no. 2, pp. 67-75, 2002.

[9] T. C. Moon, C. X. Lin, J. S. Lee, D. S. Kim, K. Bae, and K. H. Son, "Antiinflammatory activity of astilbic acid from Astilbe chinensis," in Biological \& Pharmaceutical Bulletin, vol. 28, p. 24, pp. 24-6. PubMed PMID, 15635157, 2005, PubMed PMID.

[10] B.-R. Jeon, Antithrombotic mechanisms of natural plant extracts, Kyungpook National University, Daegu, Republic of Korea, 2017.

[11] S. Sancheti, S. H. Lee, J. E. Lee, and S. Y. Seo, "Screening of Korean Medicinal Plant Extracts for alpha-Glucosidase Inhibitory Activities," Iranian journal of pharmaceutical research: IJPR, vol. 10, no. 2, Article ID 24250352, p. 261, 2011.

[12] M. Irfan, D. Jeong, E. Saba et al., " Gintonin modulates platelet function and inhibits thrombus formation ," Platelets, pp. 1-10, 2018.

[13] D. Jeong, M. Irfan, S.-D. Kim et al., "Ginsenoside Rg3-enriched red ginseng extract inhibits platelet activation and in vivo thrombus formation," Journal of Ginseng Research, vol. 41, no. 4, pp. 548-555, 2017.

[14] J. Schaeffer and M. P. Blaustein, "Platelet free calcium concentrations measured with fura- 2 are influenced by the transmembrane sodium gradient," Cell Calcium, vol. 10, no. 2, pp. 101-113, 1989.

[15] M. Irfan, T. Kwon, B. Yun, N. Park, and M. H. Rhee, "Eisenia bicyclis (brown alga) modulates platelet function and inhibits thrombus formation via impaired P 2 Y 12 receptor signaling pathway," Phytomedicine, vol. 40, pp. 79-87, 2018. 
[16] F. Adam, A. Kauskot, J.-P. Rosa, and M. Bryckaert, "Mitogenactivated protein kinases in hemostasis and thrombosis," Journal of Thrombosis and Haemostasis, vol. 6, no. 12, pp. 2007-2016, 2008.

[17] G. Altamura, B. d. Uberti, G. Galiero et al., "Expression and activation of platelet-derived growth factor $\beta$ receptor, mitogenactivated protein/extracellular signal-regulated kinase kinase (MEK) and extracellular signal-regulated kinase (ERK) in canine mammary tumours," Research in Veterinary Science, vol. 110, pp. 29-33, 2017.

[18] S. P. Jackson, S. M. Schoenwaelder, I. Goncalves et al., "PI 3kinase p110 $\beta$ : A new target for antithrombotic therapy," Nature Medicine, vol. 11, no. 5, pp. 507-514, 2005.

[19] M. Endale, W. M. Lee, S. M. Kamruzzaman et al., "GinsenosideRp1 inhibits platelet activation and thrombus formation via impaired glycoprotein VI signalling pathway, tyrosine phosphorylation and MAPK activation," British Journal of Pharmacology, vol. 167, no. 1, pp. 109-127, 2012.

[20] J. M. Gibbins, "Platelet adhesion signalling and the regulation of thrombus formation," Journal of Cell Science, vol. 117, no. 16, pp. 3415-3425, 2004.

[21] J. Y. Park, H. D. Ji, B. R. Jeon et al., "Chlorin e6 Prevents ADPInduced Platelet Aggregation by Decreasing PI3K-Akt Phosphorylation and Promoting cAMP Production," Evidence-Based Complementary and Alternative Medicine, vol. 2013, Article ID 569160, 11 pages, 2013.

[22] M.-Q. Shan, Y. Qian, S. Yu et al., "Anti-inflammatory effect of volatile oil from Schizonepeta tenuifolia on carrageenininduced pleurisy in rats and its application to study of appropriate harvesting time coupled with multi-attribute comprehensive index method," Journal of Ethnopharmacology, vol. 194, pp. 580586, 2016.

[23] M. J. Berridge, M. D. Bootman, and H. L. Roderick, "Calcium signalling: dynamics, homeostasis and remodelling," Nature Reviews Molecular Cell Biology, vol. 4, no. 7, pp. 517-529, 2003.

[24] C. Oury, E. Toth-Zsamboki, J. Vermylen, and M. F. Hoylaerts, "P2X1-mediated activation of extracellular signal-regulated kinase 2 contributes to platelet secretion and aggregation induced by collagen," Blood, vol. 100, no. 7, pp. 2499-2505, 2002.

[25] Z. M. Ruggeri, "Platelets in atherothrombosis," Nature Medicine, vol. 8, no. 11, pp. 1227-1234, 2002.

[26] D. R. Phillips, K. S. S. Prasad, J. Manganello, M. Bao, and L. Nannizzi-Alaimo, "Integrin tyrosine phosphorylation in platelet signaling," Current Opinion in Cell Biology, vol. 13, no. 5, pp. 546-554, 2001.

[27] Z. M. Ruggeri and G. L. Mendolicchio, "Adhesion mechanisms in platelet function," Circulation Research, vol. 100, no. 12, pp. 1673-1685, 2007.

[28] A. Kuliopulos, R. Mohanlal, and L. Covic, "Effect of selective inhibition of the p38 MAP kinase pathway on platelet aggregation," Thrombosis and Haemostasis, vol. 92, no. 6, pp. 1387-1393, 2004.

[29] Y. A. Senis, A. Mazharian, and J. Mori, "Src family kinases: at the forefront of platelet activation," Blood, vol. 124, no. 13, pp. 2013-2024, 2014.

[30] H. Wu, M. Ye, J. Yang et al., "Nicorandil protects the heart from ischemia/reperfusion injury by attenuating endoplasmic reticulum response-induced apoptosis through PI3K/Akt signaling pathway," Cellular Physiology and Biochemistry, vol. 35, no. 6, pp. 2320-2332, 2015.

[31] J. M. Cosemans, I. C. Munnix, R. Wetzker, R. Heller, S. P. Jackson, and J. W. Heemskerk, "Continuous signaling via PI3K isoforms beta and $\gamma$ is required for platelet ADP receptor function in dynamic thrombus stabilization," Blood, vol. 108, no. 9, pp. 3045-3052, 2006. 


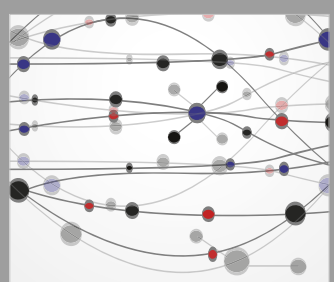

The Scientific World Journal
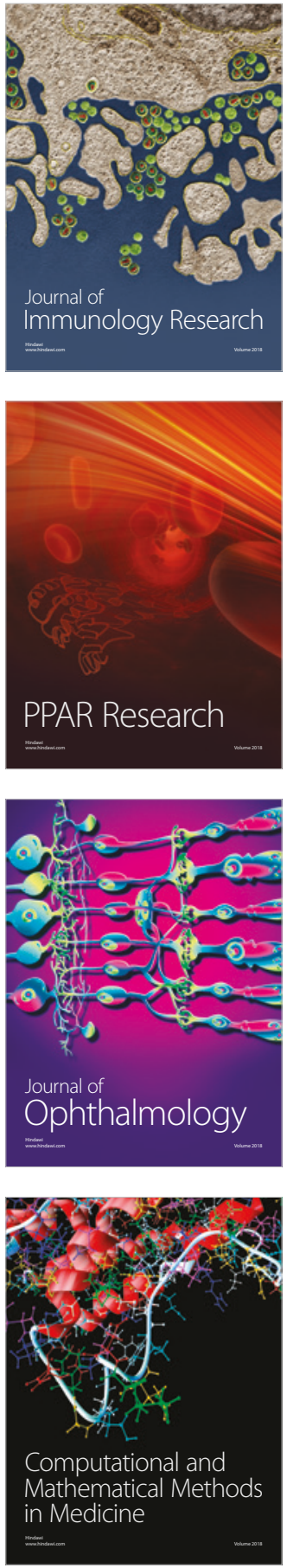

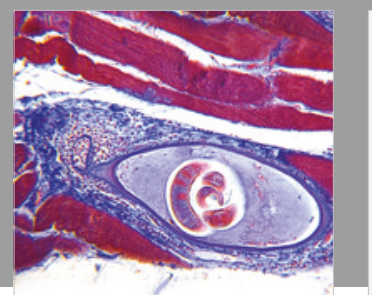

Gastroenterology Research and Practice

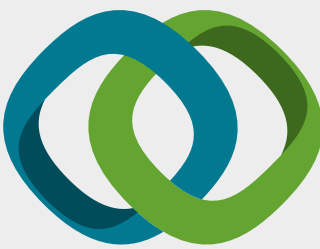

\section{Hindawi}

Submit your manuscripts at

www.hindawi.com
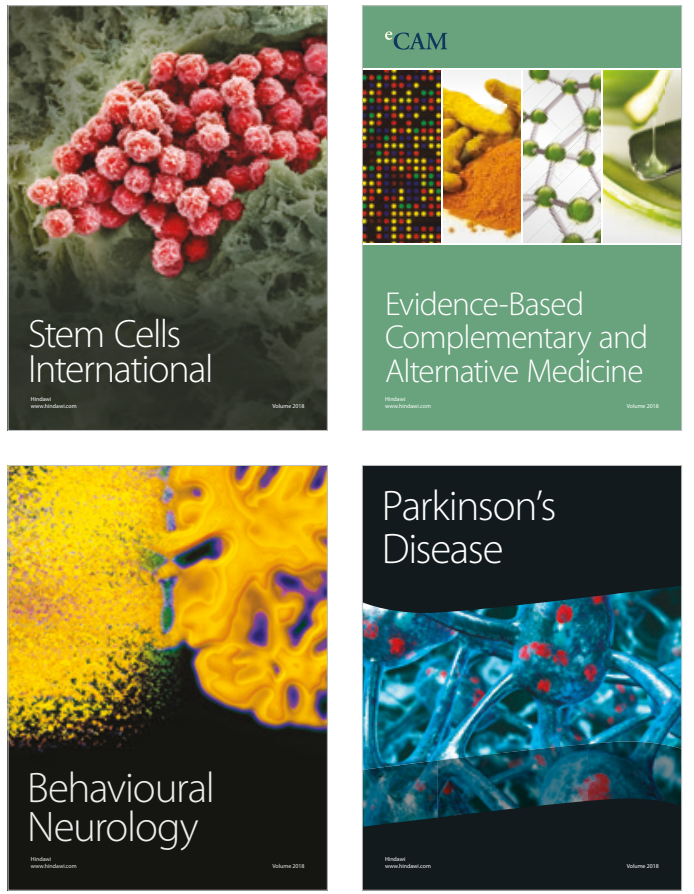

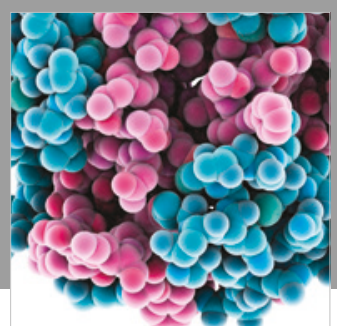

ournal of

Diabetes Research

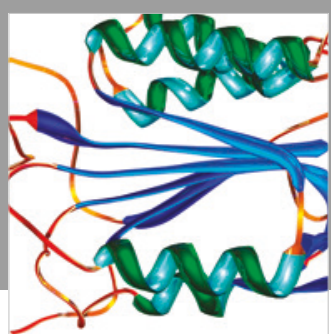

Disease Markers
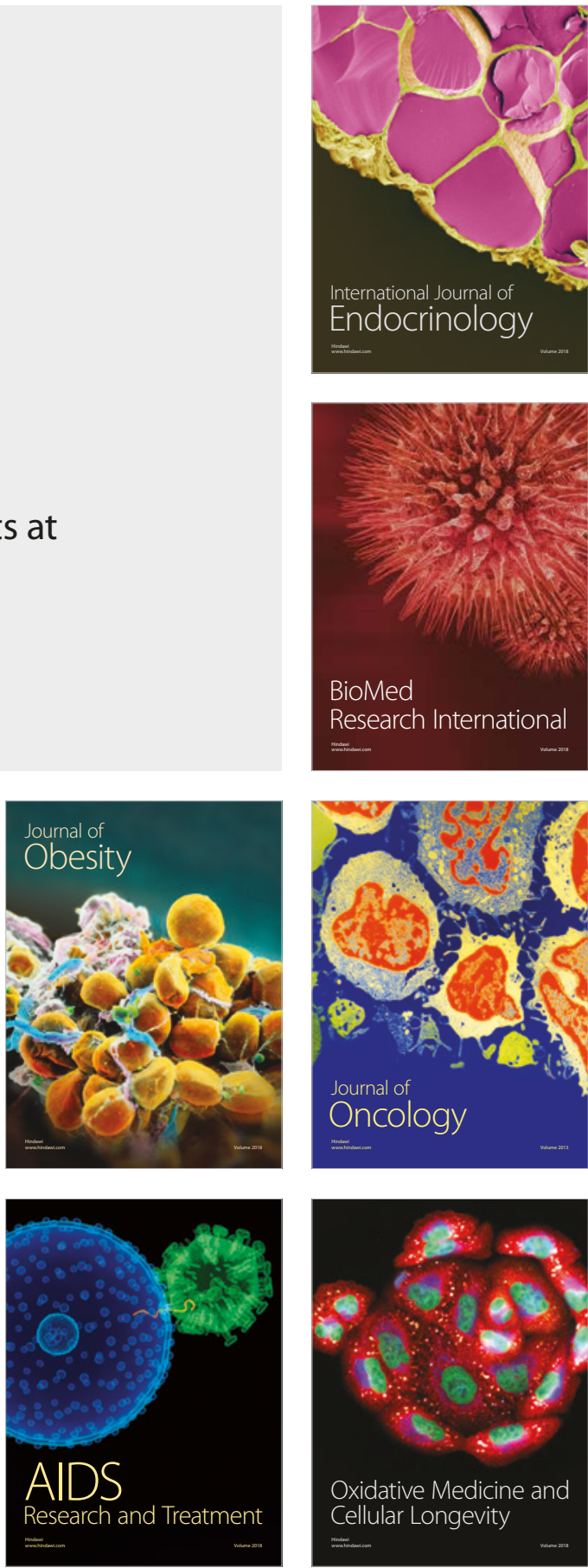